\title{
On the Expressiveness of Infinite Behavior and Name Scoping in Process Calculi
}

\author{
Pablo Giambiagi ${ }^{1}$, Gerardo Schneider $^{2,3 \star}$, and Frank D. Valencia ${ }^{3 \star \star}$ \\ 1 KTH Royal Institute of Technology, IMIT, Electrum 229, 16440 Kista, Sweden \\ pgiamb@imit.kth.se \\ 2 IRISA/CNRS, Campus de Beaulieu F-35042 Rennes, France \\ 3 Uppsala University, Dept. of Computer Systems, Box 337, 75105 Uppsala, Sweden \\ \{gerardos,frankv\}@it.uu.se
}

\begin{abstract}
In the literature there are several CCS-like process calculi differing in the constructs for the specification of infinite behavior and in the scoping rules for channel names. In this paper we study various representatives of these calculi based upon both their relative expressiveness and the decidability of divergence. We regard any two calculi as being equally expressive iff for every process in each calculus, there exists a weakly bisimilar process in the other.

By providing weak bisimilarity preserving mappings among the various variants, we show that in the context of relabeling-free and finite summation calculi: (1) CCS with parameterless (or constant) definitions is equally expressive to the variant with parametric definitions. (2) The CCS variant with replication is equally expressive to that with recursive expressions and static scoping. We also state that the divergence problem is undecidable for the calculi in (1) but decidable for those in (2). We obtain this from (un)decidability results by Busi, Gabbrielli and Zavattaro, and by showing the relevant mappings to be computable and to preserve divergence and its negation. From (1) and the well-known fact that parametric definitions can replace injective relabelings, we show that injective relabelings are redundant (i.e., derived) in CCS (which has constant definitions only).
\end{abstract}

\section{Introduction}

The study of concurrency is often conducted with the aid of process calculi. Undoubtedly CCS [9], a calculus for synchronous communication, remains a standard representative. In fact, many foundational ideas in the theory of concurrency have grown out of this calculus.

Nevertheless, there are several variants of CCS in the literature. This is reasonable as a variant may simplify the presentation of the calculus or be tailored to specific applications. Given two variants, a legitimate question is whether they are equally expressive. To answer this question one has to agree on what it means for one calculus to be as expressive as the other. A natural way of doing this in CCS is by comparing w.r.t. some standard process equivalence such as (weak) bisimilarity: If for every process $P$ in one calculus there is a process $Q$ in the other calculus such that $Q$ is (weakly) bisimilar to

\footnotetext{
* Work supported by European project ADVANCE, Contract No. IST-1999-29082.

${ }^{\star \star}$ Work supported by European project PROFUNDIS.
} 
$P$ then we say that the second calculus is at least as expressive as the first one. Another legitimate question, given a variant, is whether some fundamental property such as $d i$ vergence (i.e., the existence of divergent computations) becomes simpler or harder to analyze.

In this paper, we study both the relative expressiveness w.r.t. weak bisimilarity and the decidability of divergence for various CCS-like calculi. We shall focus upon two sources of variation found in the CCS literature: The constructs used to express infinite behavior and the way in which scoping of channel (port) names is dealt with. As for the constructs for finite behavior, in all the calculi we confine our attention to prefix, finite sums, restriction, and parallel composition. The calculi here studied can be described as follows:

- $\mathrm{CCS}_{\mathrm{k}}$ : Infinite behavior is given by a finite set of constant (i.e., parameterless) definitions of the form $A \stackrel{\text { def }}{=} P$. The calculus is essentially CCS [9] with neither relabelings nor infinite summations.

- $\mathrm{CCS}_{\mathrm{p}}$ : Like $\mathrm{CCS}_{\mathrm{k}}$ but using parametric definitions of the form $A\left(x_{1}, \ldots, x_{n}\right) \stackrel{\text { def }}{=} P$. The calculus is the variant in [10], Part I.

- $\mathrm{CCS}_{\text {! }}$ : Infinite behavior given by replication of the form $! P$. This variant is presented in [3].

- $\mathrm{CCS}_{\mu}$ : Infinite behavior given by recursive expressions of the form $\mu X . P$ as in [9]. However, we adopt static scoping of channel names in the sense discussed in [5].

In particular, we show that (1) $\mathrm{CCS}_{\mathrm{k}}$ is exactly as expressive as $\mathrm{CCS}_{\mathrm{p}}$ while (2) $\mathrm{CCS}_{\mu}$ is exactly as expressive as $\mathrm{CCS}_{\text {! }}$. We use recent work by Busi et al. [3] to also state that (3) the divergence problem is undecidable for the calculi in (1) but decidable for those in (2). The results (1-3) are summarized in Figure 1

Also, as a consequence of (1), we prove that (4) injective relabelings, from the expressiveness point of view, are redundant operators in CCS. More precisely, the behavior of any CCS process involving relabelings (all of them being injective) can be expressed up to strong bisimilarity by a $\mathrm{CCS}_{\mathrm{k}}$ process. Furthermore, we also illustrate that $\mathrm{CCS}_{\mathrm{k}}$ exhibits dynamic scoping of channel names and that it does not satisfy $\alpha$-conversion. By dynamic scoping we mean that, unlike the static case, the occurrence of a name can get dynamically (i.e., during execution) captured under a restriction.

Let us now elaborate on the significance and implications of the above results. A noteworthy aspect of (1) is that any finite set of parametric (possibly mutually recursive) definitions can be replaced by an also finite set of parameterless definitions using neither infinite summations nor relabelings. This arises as a result of the restricted nature of communication in CCS (e.g., absence of mobility). Related to this result is that of [9] which shows that, in the context of value-passing CCS, a parametric definition can be encoded using an infinite set of constant definitions and infinite sums.

Regarding (1) some readers may feel that given a process $P$ with a parametric definition $D$, one could simply create as many constant definitions as permutations of possible parameters w.r.t. the finite set of names in $P$ and $D$. This would not work for $\mathrm{CCS}_{\mathrm{p}}$; an unfolding of $D$ within a restriction may need $\alpha$-conversions to avoid name captures, thus generating new names (i.e., names not in $P$ nor $D$ ) during execution.

The interesting point about (4) is that injective relabelings are perhaps the most used kind of relabelings (e.g., injective relabelings are used in [9] to define linking operators, 

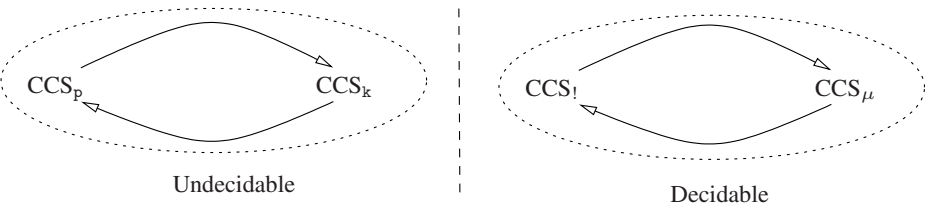

Fig. 1. Classification of CCS variants. An arrow from $X$ to $Y$ indicates that $Y$ is at least as expressive as $X$. (Un)decidability is understood w.r.t. the existence of divergent computations

buffers, counters and stacks). In fact, [9] points out that the CCS laws for equational reasoning with injective relabelings as side conditions can usually be applied as one mostly works with this kind of relabeling. In the context of SCCS, another CCS variant where interaction is synchronous, idempotent relabelings are known to be redundant [8]. In fact, under some natural assumptions, the same holds for general relabelings in SCCS.

Another noteworthy aspect of our results is the qualitative distinction between static and dynamic name scoping for the calculi under consideration. Static scoping renders the calculus decidable (w.r.t. the divergence problem) and as expressive as that with replication. In contrast, dynamic scoping renders the calculus undecidable and as expressive as that with parametric definitions. This is interesting, since as we shall see, the difference between the calculi with static or dynamic scoping is very subtle. Using static scoping for recursive expressions was discussed in the context of ECCS [5], an extension of CCS whose ideas lead to the design of the $\pi$-calculus [10].

It should be noticed that preservation of divergence is not a requirement for equality of expressiveness; weak bisimilarity does not preserve divergence. Hence, although the results in [3] prove that divergence is decidable for $\mathrm{CCS}_{\text {! }}$ (and undecidable for $\mathrm{CCS}_{\mathrm{p}}$ ), it does not follow directly from the arrows in Figure 1 that it is also decidable for $\mathrm{CCS}_{\mu}$.

Finally, it is worth pointing out that, as exposed in [7], decidability of divergence does not imply lack of Turing expressiveness. In fact the authors in [2] show that $\mathrm{CCS}_{\text {! }}$ is Turing-complete. But this does not imply that $\mathrm{CCS}_{\text {! }}$ is equally expressive to $\mathrm{CCS}_{\mathrm{p}}$ either; the notions of expressiveness used in concurrency theory may not coincide with those in computability. For example, [11] shows that under some reasonable assumptions the asynchronous version of the $\pi$-calculus, which can certainly encode Turing Machines, is strictly less expressive than the synchronous one.

Overall, the general contribution of this paper is to provide and clarify some qualitative and semantics distinctions among various CCS variants.

\section{CCS-Like Calculi}

We shall classify CCS-like calculi that differ in their way of specifying infinite behavior and name scope. Let us begin with their common finite fragment.

In CCS, processes can perform actions or synchronize on them. These actions can be either offering port names for communication, or the so-called silent action $\tau$. We presuppose a countable set $\mathcal{N}$ of port names, ranged over by $a, b, x, y \ldots$ and their 
Table 1. An operational semantics for a process calculus

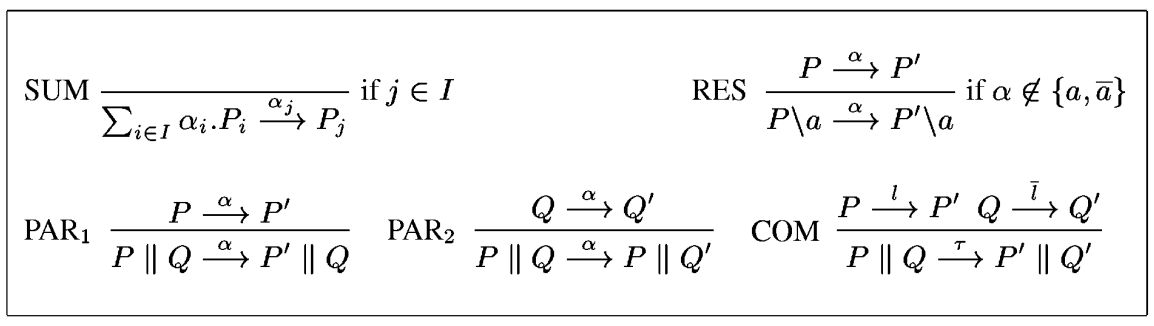

primed versions. We then introduce a set of co-names $\overline{\mathcal{N}}=\{\bar{a} \mid a \in \mathcal{N}\}$ disjoint from $\mathcal{N}$. The set of labels, ranged over by $l$ and $l^{\prime}$, is $\mathcal{L}=\mathcal{N} \cup \overline{\mathcal{N}}$. The set of actions Act, ranged over by $\alpha$ and $\beta$, extends $\mathcal{L}$ with a new symbol $\tau$. Actions $a$ and $\bar{a}$ are thought of as complementary, so we decree that $\overline{\bar{a}}=a$. We also decree that $\bar{\tau}=\tau$.

The processes specifying finite behavior are given by:

$$
P, Q \ldots::=\sum_{i \in I} \alpha_{i} . P_{i}|P \backslash a| P \| Q
$$

Intuitively $\sum_{i \in I} \alpha_{i} . P_{i}$, where $I$ is a finite set of indexes, represents a process able to perform one-but only one-of its $\alpha_{i}$ 's actions and then behave as the corresponding $P_{i}$. We write the summation as $\mathbf{0}$ if $|I|=0$, and drop the " $\sum_{i \in I}$ " if $|I|=1$. The restriction $P \backslash a$ behaves as $P$ except that it can offer neither $a$ nor $\bar{a}$ to its environment. The names $a$ and $\bar{a}$ in $P$ are said to be bound in $P \backslash a$. The bound names of $P, b n(P)$, are those with a bound occurrence in $P$, and the free names of $P, f n(P)$, are those with a not bound occurrence in $P$. Finally, $P \| Q$ represents parallelism; either $P$ or $Q$ may perform an action, or they can also synchronize when performing complementary actions.

The above description is made precise by the operational semantics in Table 1. A transition $P \stackrel{\alpha}{\longrightarrow} Q$ says that $P$ can perform $\alpha$ and evolve into $Q$.

In the literature there are at least four alternatives to extend the above syntax to express infinite behavior. We describe them next.

\subsection{Parametric Definitions: $\mathrm{CCS}_{\mathrm{p}}$}

A common way of specifying infinite behavior is by using parametric definitions [10]. In this case we extend the syntax of finite processes (Equation 1) as follows:

$$
P, Q, \ldots:=\ldots \mid A\left(y_{1}, \ldots, y_{n}\right)
$$

Here $A\left(y_{1}, \ldots, y_{n}\right)$ is an identifier (also call, or invocation) of arity $n$. We assume that every such an identifier has a unique, possibly recursive, definition $A\left(x_{1}, \ldots, x_{n}\right) \stackrel{\text { def }}{=}$ $P_{A}$ where the $x_{i}$ 's are pairwise distinct, and the intuition is that $A\left(y_{1}, \ldots, y_{n}\right)$ behaves as its body $P_{A}$ with each $y_{i}$ replacing the formal parameter $x_{i}$. We denote by $\mathcal{D}$ the set of all definitions. We often use the notation $\boldsymbol{x}$ as an abbreviation of $x_{1}, x_{2}, \ldots, x_{n}$.

Convention 1 (Finitary $\mathcal{D}$ ) Similar to [13], we shall require any process to depend only on finitely many definitions. Below we formalize this requirement. 
Given $A(\boldsymbol{x}) \stackrel{\text { def }}{=} P_{A}$ and $B(\boldsymbol{y}) \stackrel{\text { def }}{=} P_{B}$ in $\mathcal{D}$, we say that $A$ (directly) depends on $B$, written $A \leadsto B$, if there is an invocation $B(\boldsymbol{z})$ in $P_{A}$. The above requirement can be then formalized by requiring the strict order induced by $\sim^{*}$ (the reflexive and transitive closure of $\sim \sqrt{1}$ to be well-founded. We also stipulate the following requirement.

Convention 2 For each $A\left(x_{1}, \ldots, x_{n}\right) \stackrel{\text { def }}{=} P_{A}$, we require $f n\left(P_{A}\right) \subseteq\left\{x_{1}, \ldots, x_{n}\right\}$.

We shall use $\mathrm{CCS}_{\mathrm{p}}$ to denote the calculus with parametric definitions with the above syntactic restrictions. The rules for $\mathrm{CCS}_{\mathrm{p}}$ are those in Table 1 plus the rule:

$$
\mathrm{CALL} \frac{P_{A}\left[y_{1}, \ldots, y_{n} / x_{1}, \ldots, x_{n}\right] \stackrel{\alpha}{\longrightarrow} P^{\prime}}{A\left(y_{1}, \ldots, y_{n}\right) \stackrel{\alpha}{\longrightarrow} P^{\prime}} \text { if } A\left(x_{1}, \ldots, x_{n}\right) \stackrel{\text { def }}{=} P_{A}
$$

As usual $P\left[y_{1} \ldots y_{n} / x_{1} \ldots x_{n}\right]$ results from syntactically replacing every free occurrence of $x_{i}$ with $y_{i}$ renaming bound names, i.e., performing name $\alpha$-conversion, wherever needed to avoid capture. It follows from [10] that in $\mathrm{CCS}_{\mathrm{p}}$ we can identify process expressions obtained by renaming bound names (so $P \backslash a$ is the same as $P[b / a] \backslash b$ ). We then say that $\mathrm{CCS}_{\mathrm{p}}$ satisfies name $\alpha$-equivalence .

\subsection{Constant Definitions: $\mathrm{CCS}_{\mathrm{k}}$}

We now consider the alternative for infinite behavior given in CCS [9]. We refer to identifiers with arity zero and their corresponding definitions as constants and constant (or parameterless) definitions, respectively. We omit the "( )" in $A($ ).

Given $A \stackrel{\text { def }}{=} P$, requiring all names in $f n(P)$ to be formal parameters, as we did for $\mathrm{CCS}_{\mathrm{p}}$ (Convention 2), would be too restrictive $-P$ would have no visible actions. Consequently, let us drop the requirement in Convention 2 to consider a fragment allowing only constant definitions but with possible occurrence of free names in their bodies. The rules for this fragment, which we call $\mathrm{CCS}_{\mathrm{k}}$, are simply those of $\mathrm{CCS}_{\mathrm{p}}$. In this case Rule CALL (which for $\mathrm{CCS}_{\mathrm{k}}$ we prefer to call CONS) takes the form

$$
\operatorname{CONS} \frac{P_{A} \stackrel{\alpha}{\longrightarrow} P^{\prime}}{A \stackrel{\alpha}{\longrightarrow} P^{\prime}} \text { if } A \stackrel{\text { def }}{=} P_{A}
$$

i.e., no $\alpha$-conversion involved; thus allowing name captures. As illustrated in the next section, this causes scoping to be dynamic and $\alpha$-equivalence not to hold.

Relabelings. The reader familiar with process algebras may have noticed that $\mathrm{CCS}_{\mathrm{k}}$ is basically CCS except for the absence of relabeling. A relabeling $f: A c t \rightarrow A c t$ is the identity for all but finitely many actions. Furthermore, $f$ satisfies $\overline{f(a)}=f(\bar{a})$, $f(a) \neq \tau$ and $f(\tau)=\tau$. For each action $\alpha$ performed by $P$, the relabeled process $P(f)$ executes $f(\alpha)$. More precisely:

$$
\mathrm{REL} \frac{P \stackrel{\alpha}{\longrightarrow} P^{\prime}}{P(f) \stackrel{f(\alpha)}{\longrightarrow} P^{\prime}(f)}
$$

\footnotetext{
${ }^{1}$ The relation $\leadsto$ is a preorder. By induced strict order we mean the strict component of $\leadsto$ * modulo the equivalence relation obtained by taking the symmetric closure of $\sim$ * .
} 
Remark 1. It is well known that the behavior specified by any process involving only injective relabelings can be equivalently specified (up to strong bisimilarity) by a relabeling-free process with the help of parametric definitions [12]. This is important since, as pointed out in [9], one usually works with injective relabelings.

\subsection{Recursion Expressions: $\mathrm{CCS}_{\mu}$}

Hitherto we have seen process expressions whose recursive behaviors are specified by an underlying set of definitions. It is often convenient, however, to have expressions which can specify recursive behavior on their own. Let us now extend our set of finite processes (Equation 1) with such recursive expressions:

$$
P, Q, \ldots:=\ldots|X| \mu X . P
$$

Here $\mu X . P$ binds the occurrences of the process variable $X$ in $P$. As for bound and free names, we define the bound variables of $P, b v(P)$ are those with a bound occurrence in $P$, and the free variables of $P, f v(P)$ are those with a not bound occurrence in $P$. An expression generated by the above syntax is said to be a process (expression) iff it is closed (i.e., it contains no free variables). The process $\mu X . P$ behaves as $P$ with the free occurrences of $X$ replaced by $\mu X . P$ applying variable $\alpha$-conversions wherever necessary to avoid captures. The semantics $\mu X . P$ is given by the rule:

$$
\operatorname{REC} \frac{P[\mu X . P / X] \stackrel{\alpha}{\longrightarrow} P^{\prime}}{\mu X . P \stackrel{\alpha}{\longrightarrow} P^{\prime}}
$$

We call $\mathrm{CCS}_{\mu}$ the resulting calculus. From [5] it follows that in $\mathrm{CCS}_{\mu}$ we can identify processes up to name $\alpha$-equivalence. Furthermore, we make a typical assumption on $\mathrm{CCS}_{\mu}$ process variables; they need to be guarded. We say that an expression is guarded in $P$ iff it lies within some sub-expression of $P$ of the form $\alpha . Q$.

Convention 3 (Guarded Recursion) We shall confine ourselves to $C C S_{\mu}$ processes where all variables are guarded.

Static and Dynamic Scope. An interesting issue regarding expression $P[\mu X . P / X]$ (cf. rule REC) is whether bound names in $P$ should be renamed to avoid captures (i.e., name $\alpha$-conversion). Such a requirement seems necessary should we want to identify processes up to $\alpha$-equivalence. In fact, the requirement gives $\mathrm{CCS}_{\mu}$ static scoping of names. Let us illustrate this with an example.

Example 1. Consider $\mu X . P$ with $P=(a \|(\bar{a} . b \| X) \backslash a)$. First, let us assume we perform name $\alpha$-conversions to avoid captures. So, $[\mu X . P / X]$ in $P$ renames the bound $a$ by a fresh name, say $c$, thus avoiding the capture of $P^{\prime} s$ free $a$ in the replacement: I.e,

$$
P[\mu X . P / X]=(a \|(\bar{c} . b \| \mu X . P) \backslash c)=(a \|(\bar{c} . b \| \mu X .(a \|(\bar{a} . b \| X) \backslash a)) \backslash c)
$$

The reader may care to verify (using the rules in Table 1 plus Rule REC) that $b$ will not be performed; i.e., there is no $\mu X . P \stackrel{\alpha_{1}}{\longrightarrow} P_{1} \stackrel{\alpha_{2}}{\longrightarrow}$.. s.t. $\alpha_{i}=b$. 
Now let us assume that the substitution makes no name $\alpha$-conversion. This causes a free occurrence of $a$ in $P$ (indicated by the dashed circle) to get bound, dynamically, in the scope of the outermost restriction: I.e.,

$$
P[\mu X . P / X]=(a \|(\bar{a} . b \| \mu X . P) \backslash a)=(a \|(\bar{a} . b \| \mu X .(\because \ddot{a}: \|(\bar{a} . b \| X) \backslash a)) \backslash a) .
$$

The reader can verify that, in this case, $b$ may eventually be performed. Such an execution of $b$ cannot be performed by $\mu X . Q$ where $Q$ is $(a \|(\bar{c} . b \| X) \backslash c)$ i.e, $P$ with the binding and bound occurrence of $a$ syntactically replaced with $c$. This shows that name $\alpha$ equivalence does not hold when dynamic scoping is used.

Remark 2. It should be pointed out that using recursive expressions with no name $\alpha$ conversion is in fact equivalent to using instead constant definitions as in the previous calculus $\mathrm{CCS}_{\mathrm{k}}$. In fact, in presenting CCS, [9] uses alternatively both kinds of constructions: using Rule REC, with no name $\alpha$-conversion, for one and Rule CONS for the other. For example, by taking $A \stackrel{\text { def }}{=} P$ with $P$ as in Example 1 one can verify that, in $\mathrm{CCS}_{\mathrm{k}}, A$ exhibits exactly the same dynamic scoping behavior illustrated by the example. So, name $\alpha$-equivalence does not hold in CCS (exposing yet another semantic difference between CCS and the $\pi$-calculus as the latter uses static scoping and satisfies $\alpha$-equivalence).

\subsection{Replication: $\mathrm{CCS}_{\text {! }}$}

One simple way of expressing infinite behavior is by using replication. Although mostly found in calculi for mobility, replication has also been studied in the context of CCS [3, 2]. In this case the syntax of finite processes (Equation 11) is extended with:

$$
P, Q, \ldots:=\ldots \mid ! P
$$

Intuitively $! P$ behaves as $P\|P\| \ldots\|P\| ! P$; as many copies of $P$ as you wish. We call CCS! the calculus that results from the above syntax. The operational rules for $\mathrm{CCS}_{\text {! }}$ are those in Table 1 plus the following rule:

$$
\mathrm{REP} \frac{P \| ! P \stackrel{\alpha}{\longrightarrow} P^{\prime}}{! P \stackrel{\alpha}{\longrightarrow} P^{\prime}}
$$

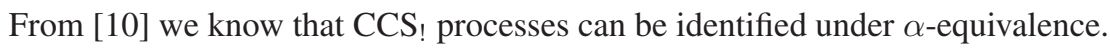

\subsection{Summary of Calculi}

We described several calculi based on the literature of CCS. We have $\operatorname{CCS}_{\mathrm{p}}$ the calculus with parametric definitions and $\mathrm{CCS}_{\mathrm{k}}$ the calculus with constant (or parameterless) definitions. We also have $\mathrm{CCS}_{\mu}$ the statically scoped calculus with recursive expressions - the dynamically scoped version instead coincides with $\mathrm{CCS}_{\mathrm{k}}$. Finally, we have the calculus with replication, $\mathrm{CCS}_{\text {! }}$. 
Convention 4 Henceforth, we use $\Sigma$ to denote the signature $\{\mathrm{p}, \mathrm{k}, \mu$, ! $\}$ of our calculi sub-indexes. We shall use $\sigma, \sigma^{\prime}, \ldots$ to range over $\Sigma$. In the following sections, we shall index sets and relations with the appropriate symbol from $\Sigma$ to make explicit the calculus under consideration. For example, $\stackrel{\alpha}{\longrightarrow}$ represents a transition of $C C S_{\sigma}$. Similarly, we shall use Proc ${ }_{\sigma}$ to denote the set of $C C S_{\sigma}$ processes. However, we may omit the indexes when these are unimportant or clear from the context.

\section{Expressiveness and Classification Criteria}

Here we introduce the means we shall use to compare and classify the various calculi.

Comparing Calculi: Bisimilarity. We wish to compare the behavior of two given processes $P$ and $Q$ w.r.t. the standard notion of (weak) bisimilarity [9]. However, $P$ and $Q$ may belong to two different calculi, say $C C S_{\sigma}$ and $C C S_{\sigma^{\prime}}$. We then find it convenient to state the standard notion as below. First, recall that the converse of a binary relation $\mathcal{S}$ is $\mathcal{S}^{-1}=\left\{\left(e^{\prime}, e\right) \mid\left(e, e^{\prime}\right) \in \mathcal{S}\right\}$

Definition 1 (Bisimilarity). A relation $\mathcal{S} \subseteq$ Proc $_{\sigma} \times$ Proc $_{\sigma^{\prime}}$, with $\sigma, \sigma^{\prime} \in \Sigma$, is said to be a (strong) simulation iff for all $(P, Q) \in \mathcal{S}$ :

$$
\text { whenever } P \stackrel{\alpha}{\longrightarrow}{ }_{\sigma} P^{\prime} \text { then, for some } Q^{\prime}, Q \stackrel{\alpha}{\longrightarrow} \sigma^{\prime} Q^{\prime} \text { and }\left(P^{\prime}, Q^{\prime}\right) \in \mathcal{S} \text {. }
$$

The relation $\mathcal{S}$ is called a (strong) bisimulation if both $\mathcal{S}$ and its converse are simulations. Furthermore, we say that $P \in \operatorname{Proc}_{\sigma}$ and $Q \in \operatorname{Proc}_{\sigma^{\prime}}$ are strongly bisimilar (w.r.t., $\sigma$ and $\left.\sigma^{\prime}\right)$, written $P \sim_{\sigma}^{\sigma^{\prime}} Q$ (or simply $P \sim Q$ ), iff there exists a bisimulation $\mathcal{S} \subseteq$ $\operatorname{Proc}_{\sigma} \times \operatorname{Proc}_{\sigma^{\prime}}$, such that $(P, Q) \in \mathcal{S}$. The relation $\sim$ is called (strong) bisimilarity.

Let us now recall the weaker notion of bisimilarity which abstracts away from silent (i.e., $\tau$ ) actions. We need some little notation. Define $\stackrel{s}{\Longrightarrow}$, with $s=\alpha_{1} \cdot \alpha_{2} \ldots \in \mathcal{L}^{*}$, as $(\stackrel{\tau}{\longrightarrow})^{*} \stackrel{\alpha_{1}}{\longrightarrow}(\stackrel{\tau}{\longrightarrow})^{*} \ldots(\stackrel{\tau}{\longrightarrow})^{*} \stackrel{\alpha_{n}}{\longrightarrow}(\stackrel{\tau}{\longrightarrow})^{*}$. The notions of weak $($ bi) simulation and weak bisimilarity can be derived from the strong versions by replacing in Definition 1 $\stackrel{\alpha}{\longrightarrow}$ and $\sim$ with $\stackrel{s}{\Longrightarrow}$ and $\approx$, respectively (cf. [9, §7.1]). We can now make precise our criterion for expressiveness.

Definition 2. We say that $C C S_{\sigma}$ is as expressive as $C C S_{\sigma^{\prime}}$ iff for every $P \in$ Proc $_{\sigma}$, there exists $Q \in \operatorname{Proc}_{\sigma^{\prime}}$ such that $P$ and $Q$ are weakly bisimilar (w.r.t. $\sigma$ and $\sigma^{\prime}$ ).

To prove equivalence on expressiveness, we shall provide (weak) bisimulation preserving mappings $\llbracket \cdot \rrbracket$, which we call encodings, from the processes of one calculus into the processes of another. Some encodings will be chosen to preserve one further property: divergence. It should be noticed that unlike strong bisimulation, weak bisimulation identifies some divergent processes with non-divergent ones. Let us formalize the notion of divergence.

Definition 3. We say that $P$ is divergent (or that it diverges) iff $P(\stackrel{\tau}{\longrightarrow})^{\omega}$, i.e., there exists an infinite sequence $P=P_{0} \stackrel{\tau}{\longrightarrow} P_{1} \stackrel{\tau}{\longrightarrow} \ldots$. 
Classifying Calculi: Decidability of Divergence. We shall classify the various calculi according to whether divergence is decidable for the calculus. By divergence being decidable for $\mathrm{CCS}_{\sigma}$, we mean that there exists an algorithm which can fully determine, given $P \in \operatorname{Proc}_{\sigma}$, whether $P$ is divergent.

\section{Encodings}

In this section we give the various encodings. Furthermore, in order to classify the calculi w.r.t. to the decidability of divergence, we shall also prove the relevant encodings to be divergence-preserving and computable.

\subsection{Encoding $\mathrm{CCS}_{\mathrm{p}}$ into $\mathrm{CCS}_{\mathrm{k}}$}

Here we give an encoding $\llbracket \cdot \rrbracket: \mathrm{CCS}_{\mathrm{p}} \rightarrow \mathrm{CCS}_{\mathrm{k}}$. For the sake of presentation, we consider only unary parametric definitions. The encoding can be easily generalized to the $n$-ary case by extending our concepts and definitions from names to vector of names.

For simplicity and w.l.o.g we assume there is a definition of the form $M^{P}(x) \stackrel{\text { def }}{=}$ $P \in D_{P}$ with $M^{P}$ not occurring in $P$ and $D_{P}$ being the finite set of definitions arising from the identifiers in $P$-think of $M^{P}$ as the "main" procedure of $P$. Formally, $D_{P}$ is the set of definitions for the identifiers in the closure under $\leadsto$ of $\left\{M^{P}\right\}$ (See Convention 11.

For the encoding we would like to associate to each process $P$ in $\operatorname{CCS}_{\mathrm{p}}$ a process in $\mathrm{CCS}_{\mathrm{k}}$ substituting $B_{y}$ for each invocation $B(y)$ in $P$. How many invocations of this form should be considered? Given that $\mathrm{CCS}_{\mathrm{p}}$ satisfies $\alpha$-equivalence, there is potentially an infinite number of such invocations - which means that a careful choice of names $y$ is needed if we want to obtain a finite number of constant definitions. To complicate things further, rule CALL may force an $\alpha$-conversion anywhere in the execution of a $\mathrm{CCS}_{\mathrm{p}}$ process.

Instead of presenting the encoding mapping right away, we proceed in a stepwise fashion. We start with the set of all $\mathrm{CCS}_{\mathrm{k}}$ processes (because of $\alpha$-conversions) that may be associated to a single $\mathrm{CCS}_{\mathrm{p}}$ process. In Def. 5. we identify sufficient conditions for subsets of those processes to define a good encoding into $\mathrm{CCS}_{\mathrm{k}}$. Finally, we show a procedure to effectively construct such an encoding.

Definition 4. The function $\widehat{\cdot}: C C S_{p} \rightarrow \mathcal{P}\left(C C S_{k}\right)$ is inductively defined over the structure of its parameter:

$$
\widehat{P}= \begin{cases}\{\mathbf{0}\} & \text { if } P=\mathbf{0} \\ \left\{\alpha \cdot Q \mid Q \in \widehat{P^{\prime}}\right\} & \text { if } P=\alpha \cdot P^{\prime} \\ \left\{Q_{1} \| Q_{2} \mid Q_{i} \in \widehat{P}_{i}, i=1,2\right\} & \text { if } P=P_{1} \| P_{2} \\ \left\{\Sigma_{i \in I} \alpha_{i} \cdot Q_{i} \mid Q_{i} \in \widehat{P}_{i}, i \in I\right\} & \text { if } P=\Sigma_{i \in I} \alpha_{i} \cdot P_{i} \\ \left\{Q \backslash \beta \mid \exists P^{\prime} \in \operatorname{Proc}_{p} \cdot P \equiv_{\alpha} P^{\prime} \backslash \beta \wedge Q \in \widehat{P^{\prime}}\right\} & \text { if } P=P^{\prime \prime} \backslash \alpha \\ \left\{A_{y}\right\} & \text { if } P=A(y) .\end{cases}
$$

Example 2. If $P=a \cdot \bar{b} \cdot \mathbf{0}+B(b)$ then $\widehat{P}$ is the singleton $\left\{a \cdot \bar{b} \cdot \mathbf{0}+B_{b}\right\}$. 
Example 3. If $P=(z . x .0\|\bar{x} .0\| A(z)) \backslash z$ then $\widehat{P}$ contains (among many others) the elements $\left(z . x .0\|\bar{x} . \mathbf{0}\| A_{z}\right) \backslash z$ and $\left(y . x .0\|\bar{x} . \mathbf{0}\| A_{y}\right) \backslash y$.

Remark 3. The definition of $\widehat{\cdot}$ is invariant under $\alpha$-conversions. More generally, it can be shown that $P \equiv_{\alpha} Q$ iff $\widehat{P}=\widehat{Q}$.

We now define $\llbracket P \rrbracket$ which requires specifying the set $\llbracket D_{P} \rrbracket$ of (constant) definitions induced by $\llbracket P \rrbracket$.

Definition 5. Given a process $P \in C C S_{p}$ with associated definition set $D_{P}$, an encoding of $P$ in $C C S_{k}$ is defined as the $C C S_{k}$ constant $M_{x}^{P}$ (called $\left.\llbracket P \rrbracket\right)$ together with an underlying set of definitions $\llbracket D_{P} \rrbracket$, satisfying the following two conditions:

(I) $\llbracket D_{P} \rrbracket$ contains a definition $\left(M_{x}^{P} \stackrel{\text { def }}{=} P_{0}\right)$ for some $P_{0} \in \widehat{P}$.

(II) If $\left(A_{y} \stackrel{\text { def }}{=} Q_{A}\right) \in \llbracket D_{P} \rrbracket, B_{z}$ occurs in $Q_{A}$ and $\left(B(x) \stackrel{\text { def }}{=} P_{B}\right) \in D_{P}$, then there is $Q_{B} \in \widehat{P_{B}[z / x]}$ s.t. $\left(B_{z} \stackrel{\text { def }}{=} Q_{B}\right) \in \llbracket D_{P} \rrbracket$.

We understand a set of definitions to contain at most one definition per process constant. A set of definitions satisfying conditions (I) and (II) is called an encoding set.

Observe that, according to the definition, there are (infinitely) many encodings for a given process $P$. Not only can an encoding be extended with definitions and still remain an encoding, but also condition (II) allows for many different definitions for constant $B_{z}$. If, say, $\left.Q_{B}, Q_{B}^{\prime} \in \widehat{P_{B}[z / x}\right]$, then an encoding $\llbracket D_{P} \rrbracket$ may contain either the definition $B_{z} \stackrel{\text { def }}{=} Q_{B}$ or the definition $B_{z} \stackrel{\text { def }}{=} Q_{B}^{\prime}$ (but not both).

The following lemma $2^{2}$ characterizes the shape of minimal encoding sets.

Lemma 1. Given an encoding set $\llbracket D_{P} \rrbracket$, the set $\mathbf{D}=\left\{\left(A_{y} \stackrel{\text { def }}{=} Q_{A}\right) \in \llbracket D_{P} \rrbracket \mid\right.$ $Q_{A} \in \widehat{\left.P_{A}[y / x]\right\}}$, is an encoding set (included in $\left.\llbracket D_{P} \rrbracket\right)$.

Recall that $D_{P}$ contains finitely many definitions. We shall show that an encoding can be effectively constructed (so that the resulting set of definitions $\llbracket D_{P} \rrbracket$ is also finite). First let us illustrate the construction with the following example.

Example 4. Let $P=A(x)$ with $D_{P}=\{A(x) \stackrel{\text { def }}{=}(z . x .0\|\bar{x} .0\| A(z)) \backslash z\}$. We proceed to define an encoding by constructing a set $\llbracket D_{P} \rrbracket$ so that it satisfies conditions (I) and (II). To satisfy condition (I), let $M_{x}^{P} \stackrel{\text { def }}{=}\left(z . x .0\|\bar{x} . \mathbf{0}\| A_{z}\right) \backslash z \in \llbracket D_{P} \rrbracket$. Then, condition (II) requires a definition such as: $A_{z} \stackrel{\text { def }}{=}\left(z_{1} . z .0\|\bar{z} . \mathbf{0}\| A_{z_{1}}\right) \backslash z_{1} \in \llbracket D_{P} \rrbracket$. Notice that due to $\alpha$-conversion in equation $A_{z}$ we have obtained a new name $z_{1}$ and hence we have to give a new definition for $A_{z_{1}}$. Of course because of the $\alpha$-conversion we could have chosen another fresh name $z_{2}$, but that would only lead to a different but equally useful encoding. Using condition (II) again: $A_{z_{1}} \stackrel{\text { def }}{=}\left(z . z_{1} . \mathbf{0}\left\|\overline{z_{1}} . \mathbf{0}\right\| A_{z}\right) \backslash z \in$ $\llbracket D_{P} \rrbracket$, and we are done; no other definition needs to be added to $\llbracket D_{P} \rrbracket$. It is easy to check that the resulting set satisfies conditions (I) and (II), and therefore constitutes an encoding of $P$ in $\mathrm{CCS}_{\mathrm{k}}$.

\footnotetext{
${ }^{2}$ See [6] for the proof of the lemmas in this paper.
} 
We now show that for any $P$, one can compute an encoding set $\llbracket D_{P} \rrbracket$.

Theorem 1. For any $P \in C C S_{p}$ with a finite set $D_{P}$ of associated definitions, one can effectively construct an encoding set $\llbracket D_{P} \rrbracket$.

Proof. Let $\operatorname{Var}\left(D_{P}\right)$ be the set of all the names occurring in $D_{P}$. For each $A(x) \stackrel{\text { def }}{=} P_{A} \in$ $D_{P}$ and each $y \in \operatorname{Var}\left(D_{P}\right)$, choose a $P_{A}^{y}$ so that $\left.P_{A}^{y} \in \widehat{P_{A}[y / x}\right]$. Define $S=\left\{A_{y} \stackrel{\text { def }}{=}\right.$ $\left.P_{A}^{y} \mid\left(A(x) \stackrel{\text { def }}{=} P_{A}\right) \in D_{P} \wedge y \in \operatorname{Var}\left(D_{P}\right)\right\}$. Notice that $S$ is a finite set. Proceed by defining $\mathcal{F}=\left\{z \mid \exists\right.$ constant $B_{z} . B_{z}$ occurs in $S \wedge B_{z}$ is not defined in $\left.S\right\}$, and notice that $\mathcal{F}$ is a finite set too. Observe that, for each definition $A(x) \stackrel{\text { def }}{=} P_{A} \in D_{P}$ and for each $y \in \mathcal{F}$, the substitution $P_{A}[y / x]$ requires no alpha-conversion. Consequently it is possible to choose $P_{A}^{y} \in \widehat{P_{A}[y / x]}$ so that for each constant $B_{z}$ occurring in $P_{A}^{y}$, $z \in\left(\operatorname{Var}\left(D_{P}\right) \cup \mathcal{F}\right)$. We have now a candidate $\Sigma_{D_{P}}$ for the set of definitions in the encoding of $P$. It is simply defined as $\Sigma_{D_{P}}=\left\{A_{y} \stackrel{\text { def }}{=} P_{A}^{y} \mid\left(A(x) \stackrel{\text { def }}{=} P_{A}\right) \in\right.$ $\left.D_{P} \wedge y \in\left(\operatorname{Var}\left(D_{P}\right) \cup \mathcal{F}\right)\right\}$. Since $\left(M_{x}^{P} \stackrel{\text { def }}{=} P_{0}\right) \in S \subseteq \Sigma_{D_{P}}$, with $P_{0} \in \widehat{P}$, our candidate set satisfies condition (I) in Def. 5. It remains to be shown that $\Sigma_{D_{P}}$ also satisfies condition (II). Assume now that $\left(A_{y} \stackrel{\text { def }}{=} Q_{A}\right) \in \Sigma_{D_{P}}$, that $B_{z}$ occurs in $Q_{A}$ and that $\left(B(x) \stackrel{\text { def }}{=} P_{B}\right) \in D_{P}$. By construction, $z \in\left(\operatorname{Var}\left(D_{P}\right) \cup \mathcal{F}\right)$, and therefore $\left(B_{z} \stackrel{\text { def }}{=} P_{B}^{z}\right) \in \Sigma_{D_{P}}$. This shows that $\Sigma_{D_{P}}$ satisfies condition (II). Therefore, our effectively constructed candidate $\Sigma_{D_{P}}$ is indeed an encoding $\llbracket D_{P} \rrbracket$.

We now state the correctness of the encoding up to (strong) bisimilarity. The theorem actually says that parametric definitions are not more expressive than constant definitions.

Theorem 2. Given a process $P \in C C S_{p}$ with associated set of definitions $D_{P}$, any encoding $\llbracket P \rrbracket$ with definition set $\llbracket D_{P} \rrbracket$ satisfies $P \sim_{p}^{k} \llbracket P \rrbracket$.

Remark 4. It follows from Remark 1 and the above theorem that injective relabelings are redundant in CCS (up to strong bisimilarity).

Now, [3] shows that divergence is undecidable for $\mathrm{CCS}_{\mathrm{p}}$. Furthermore, we also showed that the above encoding is computable. Since divergence is invariant under strong bisimilarity, we can then conclude the following result.

Theorem 3. The divergence problem is undecidable for $C C S_{k}$.

\subsection{Encoding $\mathrm{CCS}_{\mathrm{k}}$ into $\mathrm{CCS}_{\mathrm{p}}$}

Intuitively, if the free names are treated dynamically, then they could equivalently be passed as parameters. Thus, we can define the encoding as follows:

Definition 6. Given $P \in C C S_{k}$ with a set of associated constant definitions of the form $A \stackrel{\text { def }}{=} P_{A}$ and given a strict total order over names, the encoding of $P$ into $C C S_{p}$ is a process $\llbracket P \rrbracket$ with associated set of definitions

$$
\left\{A\left(x_{1}, \ldots, x_{n}\right) \stackrel{\text { def }}{=} \llbracket P_{A} \rrbracket \mid\left(A \stackrel{\text { def }}{=} P_{A}\right) \in D p \wedge f n\left(P_{A}\right)=\left\{x_{1}, \ldots, x_{n}\right\}\right\} .
$$


The encoding function $\llbracket \cdot \rrbracket:$ Proc $_{k} \rightarrow$ Proc $_{p}$, which is an homomorphism over all other operators, satisfies $\llbracket A \rrbracket=A\left(x_{1}, \ldots, x_{n}\right)$ where $f n\left(P_{A}\right)=\left\{x_{1}, \ldots, x_{n}\right\}$. Both in definitions and in invocations, all lists of argument names are assumed sorted.

(By homomorphism we mean that $\llbracket P\|Q \rrbracket=\llbracket P \rrbracket\| \llbracket Q \rrbracket$ and similarly for the other operators.)

The following theorem states that constant definitions with dynamic scoping are not more expressive than parametric definitions with static scoping.

Theorem 4. For every process $P$ in $C C S_{k}, \llbracket P \rrbracket \sim_{k}^{p} P$.

\subsection{Encoding $\mathrm{CCS}_{\mu}$ into $\mathrm{CCS}_{\text {! }}$}

The main idea behind this encoding is to associate a replicated process $! x \cdot P^{\prime}$ to each occurrence of the recursion operator, $\mu X$.P. In the past a similar approach has been used to show that, in the $\pi$-calculus, recursion can be expressed using replication [13]. While in [13] each $\pi$-calculus process and its encoding happen to be strongly bisimilar, this is not the case for $\mathrm{CCS}_{\mu}$. Although in general a $\mathrm{CCS}_{\mu}$ process is only weakly bisimilar to its encoding, we show that divergence properties are always preserved.

Our definition assumes that process variables are indexed by $I$, i.e. $\left\{X_{i} \mid i \in I\right\}$ :

Definition 7. Let $\llbracket \cdot \rrbracket:$ Proc $_{\mu} \rightarrow$ Proc! $_{\text {b }}$ be the encoding function that is homomorphic over all operators in the sub-calculus defining finite behavior and is otherwise defined as follows:

$$
\begin{aligned}
& \llbracket X_{i} \rrbracket=\overline{x_{i}} \cdot \mathbf{0} \\
& \llbracket \mu X_{i} \cdot P \rrbracket=\left(! x_{i} \cdot \llbracket P \rrbracket \| \overline{x_{i}} \cdot \mathbf{0}\right) \backslash x_{i}
\end{aligned}
$$

where the names $\left\{x_{i} \mid i \in I\right\}$ are fresh.

The freshness condition on the variables $x_{i}$ is meant to guarantee that every time we apply $\llbracket P \rrbracket, P$ mentions none of them.

Remark 5. The above encoding would not work had we adopted dynamic scoping in the Rule REC for $\mathrm{CCS}_{\mu}$ (see Remark 2). The $\mu X . P$ in Example11 actually gives us a counter-example.

The following example illustrates why a $\mathrm{CCS}_{\mu}$ process may not be strongly bisimilar to its encoding.

Example 5. Consider the $\mathrm{CCS}_{\mu}$ process $P=\mu X . a . X$ with corresponding encoding $\llbracket P \rrbracket=(! x . a . \bar{x} \| \bar{x}) \backslash x$. They are clearly not strongly bisimilar, as $P$ has the single trace $\mu X . a . X \stackrel{a}{\rightarrow}_{\mu} \mu X . a . X \stackrel{a}{\rightarrow}_{\mu} \mu X . a . X \ldots$ while $\llbracket P \rrbracket$ only produces $(! x . a . \bar{x} \| \bar{x}) \backslash x \stackrel{\tau}{\rightarrow}_{\mu}$ $(! x . a . \bar{x} \| a . \bar{x}) \backslash x \stackrel{a}{\rightarrow}_{\mu}(! x . a . \bar{x} \| \bar{x}) \backslash x \stackrel{\tau}{\rightarrow}_{\mu} \ldots$ Observe that each transition in the first trace uses rule REC, and that every other step in the second one reflects explicitly, as an internal transition, each recursive call.

In comparing $\mathrm{CCS}_{\mu}$ and $\mathrm{CCS}_{\text {! }}$, we find it convenient to consider yet another variant calculus, as an intermediate step, which we call $\mathrm{CCS}_{\tau}$ : Its syntax agrees entirely with 
$\mathrm{CCS}_{\mu}$ 's (i.e. $\operatorname{Proc}_{\tau}=\operatorname{Proc}_{\mu}$ ), and its semantics differs from $\mathrm{CCS}_{\mu}$ 's only by a replacement of REC with a rule in which the unfolding performs a $\tau$ action-hence the name $\mathrm{CCS}_{\tau}$ :

$$
\mathrm{REC}^{\prime} \quad \overline{\mu X . P \stackrel{\tau}{\rightarrow}_{\tau} P[\mu X . P / X]}
$$

Example 6. Consider process $P$ as given in Example 5 but this time within $\mathrm{CCS}_{\tau}$ (which is possible thanks to $\operatorname{Proc}_{\tau}=\operatorname{Proc}_{\mu}$ ). The only trace exhibited by $P$ is: $\mu X . a . X \stackrel{\tau}{\rightarrow}_{\tau}$ $a .(\mu X . a . X) \stackrel{a}{\rightarrow}_{\tau} \mu X . a . X \stackrel{\tau}{\rightarrow}_{\tau} \ldots$ and therefore $P \sim_{\tau}^{!} \llbracket P \rrbracket$.

In fact, the property illustrated by the previous example holds in general, as stated in the following theorem. The proof is essentially an adaptation of the one given by Sangiorgi and Walker in [13].

Theorem 5. If $P \in C C S_{\tau}$, then $P \sim_{\tau}^{!} \llbracket P \rrbracket$.

Because strong bisimilarity is known to preserve expressiveness and divergence, the above theorem lets us reduce the problem of studying the encoding to investigating the relation between $\mathrm{CCS}_{\tau}$ and $\mathrm{CCS}_{\mu}$.

We define a binary relation $\mathcal{R} \in\left(\operatorname{Proc}_{\mu} \times \operatorname{Proc}_{\tau}\right)$ as follows: $P \mathcal{R} Q$ iff there exist $n \geq 0$ such that $P=Q_{0} \stackrel{\tau}{\rightarrow}_{\tau} Q_{1} \stackrel{\tau}{\rightarrow}_{\tau} \ldots Q_{n}=Q$, where each derivation $Q_{i} \stackrel{\tau}{\rightarrow}_{\tau} Q_{i+1}$ involves the application of rule REC'.

We show that besides being a weak bisimulation relation, $\mathcal{R}$ also relates processes with equal divergence properties. As a first step, notice that each $\stackrel{\alpha}{\rightarrow}_{\mu}$ transition can be mimicked by $\mathcal{R}$-related processes in $\operatorname{CCS}_{\tau}$ after possibly some $\tau$ transitions (which correspond to recursive invocations involving rule REC').

Lemma 2. If $P \mathcal{R} Q$ and $P \stackrel{\alpha}{\rightarrow}_{\mu} P^{\prime}$ then there exists $Q^{\prime}$ such that $Q\left(\stackrel{\tau}{\rightarrow}_{\tau}\right)^{*} \stackrel{\alpha}{\rightarrow}_{\tau} Q^{\prime}$ and $P^{\prime} \mathcal{R} Q^{\prime}$.

Remark 6. Notice that we have restricted our attention to processes where all variables are guarded. Without this assumption divergence would not be preserved by our encoding. For example, $\mu X . X$ diverges in $\mathrm{CCS}_{\tau}$ but deadlocks in $\mathrm{CCS}_{\mu}$.

Lemma 3. If $P \mathcal{R} Q$ and there is a derivation of $Q \stackrel{\alpha}{\rightarrow}{ }_{\tau} Q^{\prime}$ which does not involve the application of rule REC', then there exists $P^{\prime}$ s.t. $P \stackrel{\alpha}{\rightarrow}_{\mu} P^{\prime}$ and $P^{\prime} \mathcal{R} Q^{\prime}$.

To show that two identical processes, interpreted in $\mathrm{CCS}_{\mu}$ and resp. $\mathrm{CCS}_{\tau}$, are weakly bisimilar we need to show two simulations: One is provided by Lemma2 2 and the other follows by a combination of Lemma 3 and the definition of $\mathcal{R}$ (to cover the case in which $Q \stackrel{\alpha}{\rightarrow} Q^{\prime}$ does use rule REC'). The result is summarized by our next theorem.

Theorem 6. Given a process $P$ in $C C S_{\mu}, P \approx_{\mu}^{\tau} P$.

Observe that this is still not enough to show that $\mathcal{R}$ relates processes with the same divergence properties. If $P \mathcal{R} Q$ and $Q$ diverges, Lemma 3 is not strong enough to show that $P$ may execute a single $\tau$ transition. However, it turns out that $Q$ cannot diverge by executing only recursive calls (again, a result of our assumptions on guarded summation 
and guarded recursion; see Remark 6 and [6]). So, if after some finite execution trace, $Q$ performs a $\tau$ transition that does not involve REC', we can apply Lemma 3 to deduce that $P$ may also perform a $\tau$ transition. Since this process can be repeated endlessly it must be concluded that divergence in $\mathrm{CCS}_{\tau}$ forces divergence in $\mathrm{CCS}_{\mu}$. The converse is an easy consequence of Lemma2 That is, we have shown:

Proposition 1. For $P \in C C S_{\mu}, P\left(\stackrel{\tau}{\rightarrow}_{\mu}\right)^{\omega}$ iff $P\left(\stackrel{\tau}{\rightarrow}_{\tau}\right)^{\omega}$.

Our journey from $\mathrm{CCS}_{\mu}$ to $\mathrm{CCS}_{\text {! }}$ through $\mathrm{CCS}_{\tau}$ has rendered the following result.

Corollary 1. For $P \in \operatorname{Proc}_{\mu}, P \approx_{\mu}^{!} \llbracket P \rrbracket$. Moreover, $P$ diverges iff $\llbracket P \rrbracket$ diverges.

From the above corollary, the fact that the encoding is computable, and the result of

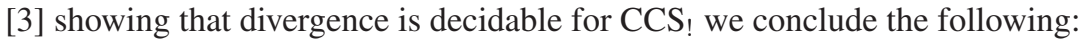

Theorem 7. The divergence problem is decidable for $C C S_{\mu}$.

\subsection{Encoding $\mathrm{CCS}_{\text {! into }} \mathrm{CCS}_{\mu}$}

Except for the syntax and our restriction to guarded recursion, this encoding is essentially that given in [13] for the $\pi$-calculus.

Definition 8. Let $\llbracket \cdot \rrbracket: P r o c ! \rightarrow P r o c_{\mu}$ be the encoding function that is homomorphic over all operators in the sub-calculus defining finite behavior and is otherwise defined as follows: $\llbracket ! P \rrbracket=\mu X .(\llbracket P \rrbracket \| \tau . X)$.

In fact, the proof of the following theorem follows that in [13].

Theorem 8. For $P \in$ Proc $_{!}, P \approx_{!}^{\mu} \llbracket P \rrbracket$.

Observe that, because of our restriction to guarded recursion, the encoding does not preserve divergence. For instance, if $P=! 0$ then $P$ is deadlocked in $\mathrm{CCS}_{\text {! }}$; but

$$
\llbracket P \rrbracket=\mu X .(\mathbf{0} \| \tau . X) \stackrel{\tau}{\rightarrow}_{\mu} \mathbf{0}\left\|\mu X .(\mathbf{0} \| \tau . X) \stackrel{\tau}{\rightarrow}_{\mu} \mathbf{0}\right\| \mathbf{0} \| \mu X .(\mathbf{0} \| \tau . X) \stackrel{\tau}{\rightarrow}_{\mu} \ldots .
$$

\section{Concluding Remarks}

We studied the relative expressiveness (w.r.t. weak bisimilarity) and the decidability of divergence for some CCS-like calculi. The calculi differ on the constructs used to express infinite behavior and on the treatment of scoping of channel names; the finite core being the same. We showed that parameters can be removed from recursive definitions without loss of expressiveness provided dynamic name scoping is applied. We also showed that the expressiveness of recursive expressions with static scoping corresponds precisely to that of replication. We partitioned the calculi into two groups: For one, divergence is undecidable (i.e., constant and parametric definitions), whereas it is decidable for the other (i.e., replication and recursive expressions with static scoping). Figure 1 in the Introduction, illustrates these results.

As a consequence of our results, we proved that a substantial family of relabelings, the injective ones, is redundant in CCS (see Remark 4). We also showed that a slightly different interpretation of Rule REC, namely performing also name $\alpha$-conversions in substitutions, can render decidable (w.r.t. divergence) an otherwise undecidable calculus (see Remark 2). We illustrated that CCS exhibits dynamic name scoping and that it does not preserve $\alpha$-equivalence. 
Related Work. Most of the related work was already discussed in the Introduction. The most closely related work is [3] which shows the (un)decidability of divergence for $\mathrm{CCS}_{\mathrm{p}}$ and $\mathrm{CCS}_{\text {! }}$. Here we extend these results to the corresponding equally expressive calculi. The work on ECCS [5], perhaps the most immediate predecessor of the $\pi$-calculus, advocates static scoping of names. In contrast, the work on CHOCS [14] advocates dynamic name scoping in the context of higher-order CCS. Furthermore, the CCS variant in [10] uses statically scoped parametric definitions while the Edinburgh Concurrency Workbench tool [4] uses dynamic scoping for parametric definitions.

The work in [1] shows that that in CCS, non-injective relabelings lead to a sensible different treatment of asynchrony w.r.t the injective ones. We believe that it would be interesting to investigate more qualitative distinctions for these two kinds of relabelings.

Acknowledgments. We are indebted to Maurizio Gabbrielli, Jean-Jacques Lévy, Sergio Maffeis, Catuscia Palamidessi, Joachim Parrow, Rosario Pugliese and Davide Sangiorgi, for insightful discussions on the topics of this paper.

\section{References}

1. M. Boreale, R. De Nicola, and R. Pugliese. Trace and testing equivalence on asynchronous processes. Information and Computation, 172(2):139-164, 2002.

2. N. Busi, M. Gabbrielli, and G. Zavattaro. The expressive power of replication in CCS. Draft, 2003.

3. N. Busi, M. Gabbrielli, and G. Zavattaro. Replication vs. recursive definitions in channel based calculi. In ICALP'03, volume 2719 of LNCS, pages 133-144. Springer Verlag, 2003.

4. R. Cleaveland, J. Parrow, and B. Steffen. The Concurrency Workbench: A semantics based tool for the verification of concurrent systems. ACM Transactions on Programming Languages and Systems, 15(1):36-72, 1993.

5. U. Engberg and M. Nielsen. A calculus of communicating systems with label-passing. Technical report, University of Aarhus, 1986.

6. P. Giambiagi, G. Schneider, and F.D. Valencia. On the expressiveness of CCS-like calculi. Technical report, Uppsala University, 2004. Postscript available from http://www.sics.se/fdt/publications/GSV-Expr-TR04.ps.

7. S. Maffeis and I. Phillips. On the computational strength of pure ambient calculi. In $E X$ PRESS'03, 2003.

8. R. Milner. Calculi for synchrony and asynchrony. Technical Report CSR-104-82, University of Edinburgh, 1982.

9. R. Milner. Communication and Concurrency. Prentice Hall, 1989.

10. R. Milner. Communicating and Mobile Systems: the $\pi$-calculus. Cambridge University Press, 1999.

11. C. Palamidessi. Comparing the expressive power of the synchronous and the asynchronous $\pi$-calculus. In ACM Press, editor, POPL'97, pages 256-265, 1997.

12. J. Parrow. An introduction to the $\pi$-calculus. In Handbook of Process Algebra, pages 479-543. Elsevier, 2001.

13. D. Sangiorgi and D. Walker. The $\pi$-calculus: A Theory of Mobile Processes. Cambridge University Press, 2001.

14. B. Thomsen. A calculus of higher order communicating systems. In POPL' 89 , ACM, pages 143-154, 1989. 